\title{
The CALMA project: a CAD tool in breast radiography
}

\author{
S.R. Amendolia, M.G. Bisogni, U. Bottigli, A. Ceccopieri, P. Delogu, A. Marchi, V.M. \\ Marzulli, R. Palmiero, S. Stumbo
}

Dipartimento di Fisica dell'Università and Sezione INFN, Pisa, Italy

In this paper we present the CALMA Project (Computer Aided Library for MAmmography), i.e. an automated search for the mammograms' texture, the massive lesions and microcalcifications clusters. CALMA's main purpose is to collect a database of mammographic images, developing CAD tools to be used as a second radiologist in the classification of breast cancer diseases. In this moment 2900 images are already in our database, which is, to our knowledge, the largest in Europe. One third of our digitized images are pathological, and they are fully characterized by a consistent description and diagnosis. We try to perform automatically the classification of mammographic images on the bases of tissues' textures. Such a classification should be done in an unbiased way with respect to radiologists and should support their interpretation of the mammographic image. Results obtained with non supervised neural networks are shown, as well as results coming from a mixed approach (features extraction combined with FF-ANN). Massive lesions are rather large objects to be detected, but they show up with a faint contrast slowly increasing with time. The need for tools able to recognize such a lesion at an early stage is therefore apparent. Our tools are based on a ROI hunter procedure for spiculated lesions showing a number of false positives of the order of 1.4 per image and keeping a $85 \%$ sensitivity on our sample. A microcalcification is a rather small ( 0.1 to $1.0 \mathrm{~mm}$ in diameter) but very brilliant. Some of them, either grouped in cluster or isolated may indicate the presence of a tumor. Up to now only 40 images with microcalcications from our database have been analyzed, and a CAD tool has been designed to detect clusters, reaching a correct classification of $90 \%$.

\section{Introduction}

Traditional mammography, using screen and film, is widely recognized as the only imaging modality for the early detection of breast cancer in asymptomatic women [1]. In this way, it has been shown to reduce significantly breast cancer mortality. Currently this accounts for $32 \%$ of cancer incidence and $18 \%$ of cancer mortality for women in the United States. 5 years survival rates are 93\% for localized cancers, falling down for regional (72\%) and distant diseases $(18 \%)$. This shows how the early detection of breast cancer is the main element in the reduction of breast cancer mortality. Typically, sensitivity ranges from $73 \%$ to $88 \%$, while specificity are of the order of $83 \%$ to $96 \%$. These relevant figures are shown to increase of $2 \%-10 \%$ if the image is analyzed independently by two radiologists [2]. The main purpose of the CALMA (Computer Aided Library for MAmmography) Collaboration is to collect a database of mammographic images, developing CAD tools to be used as a second radiologist in the classification of breast diseases. This goal can be reached performing an automated search for the mammograms' texture, the massive lesions and microcalcifications clusters.

\section{Materials and Methods}

Images come from the collaborating hospitals where mammogram films $\left(18 \times 24 \mathrm{~cm}^{2}\right)$ are scanned, using a CCD linear scanner with a 85 microns granularity and 4096 gray levels, and transferred to CD-ROM. Each image is made of about 6 million pixels corresponding to more 
than 10 Mbytes of disk space. Given the average dimensions of the images, without any compression, about 60 images can be written on a single CD-ROM. CDs are hosted in a jukebox controlled by a "Origin 200" Silicon Graphics server. The jukebox can contain up to 240 CDROMS, with an average access time of 3 seconds. In this moment 2900 images, are already in our database, which is, at our knowledge, the largest in Europe (fig. 1). One third of our digitized images are pathological, and they are fully characterized by a consistent description and diagnosis.
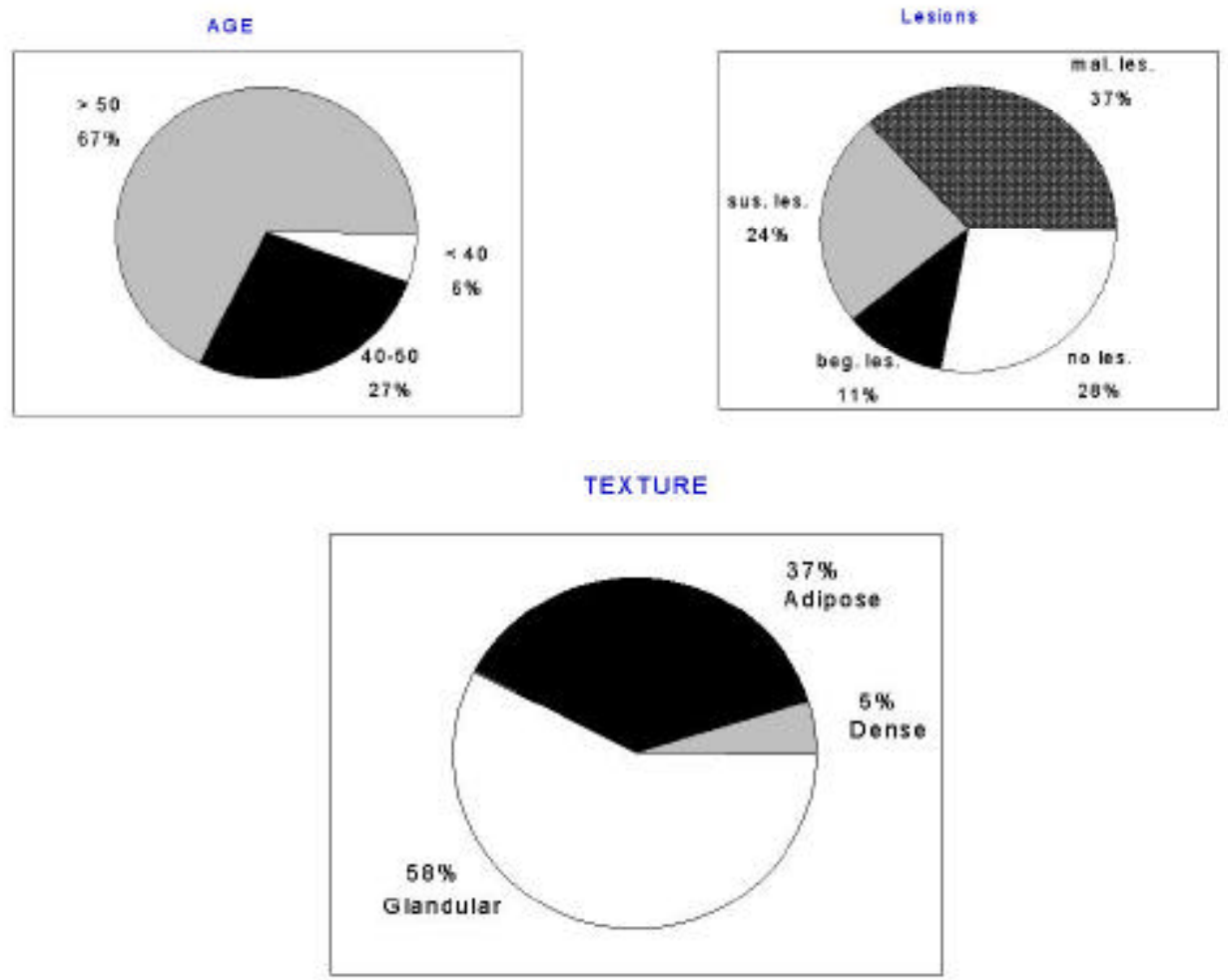

Figure 1 - CALMA database with distributions on age, lesions and textures

Typically an early breast cancer diagnosis follows from the detection of a cluster of microcalcifications. As microcalcifications may show up late in the development of the disease, a way to attack earlier the problem would be to increase the capability of detecting tumors masses characterized by a very low contrast, but peculiar shapes. At this level our strategy is developed in two steps:

The first step provides a series of tools (standard and/or neural) to choose one or more regions of the mammogram, i.e. a ROI hunter procedure. These algorithms perform standard operations on the images, such as enhancement, filters of various kinds, gradient patterns etc., as well as more dedicated operations which have been thought to cope the peculiar shapes of malignant objects.

The second step, using the neural network approach, detects the existence and the characteristics, inside of the ROI, of the lesions. 


\section{Texture analysis}

We also try to perform automatically the classification of mammographic images on the bases of tissues' textures. Such a classification should be done in an unbiased way with respect to radiologists and should support their interpretation of the mammographic image. In figure 2 two images show the same type of lesion on different backgrounds: Adipose and Glandular. In the first case the lesion appears clearly, in the second disappear in background noise produced by the glandular pattern. Under this point of view, texture analysis has a fundamental rule.
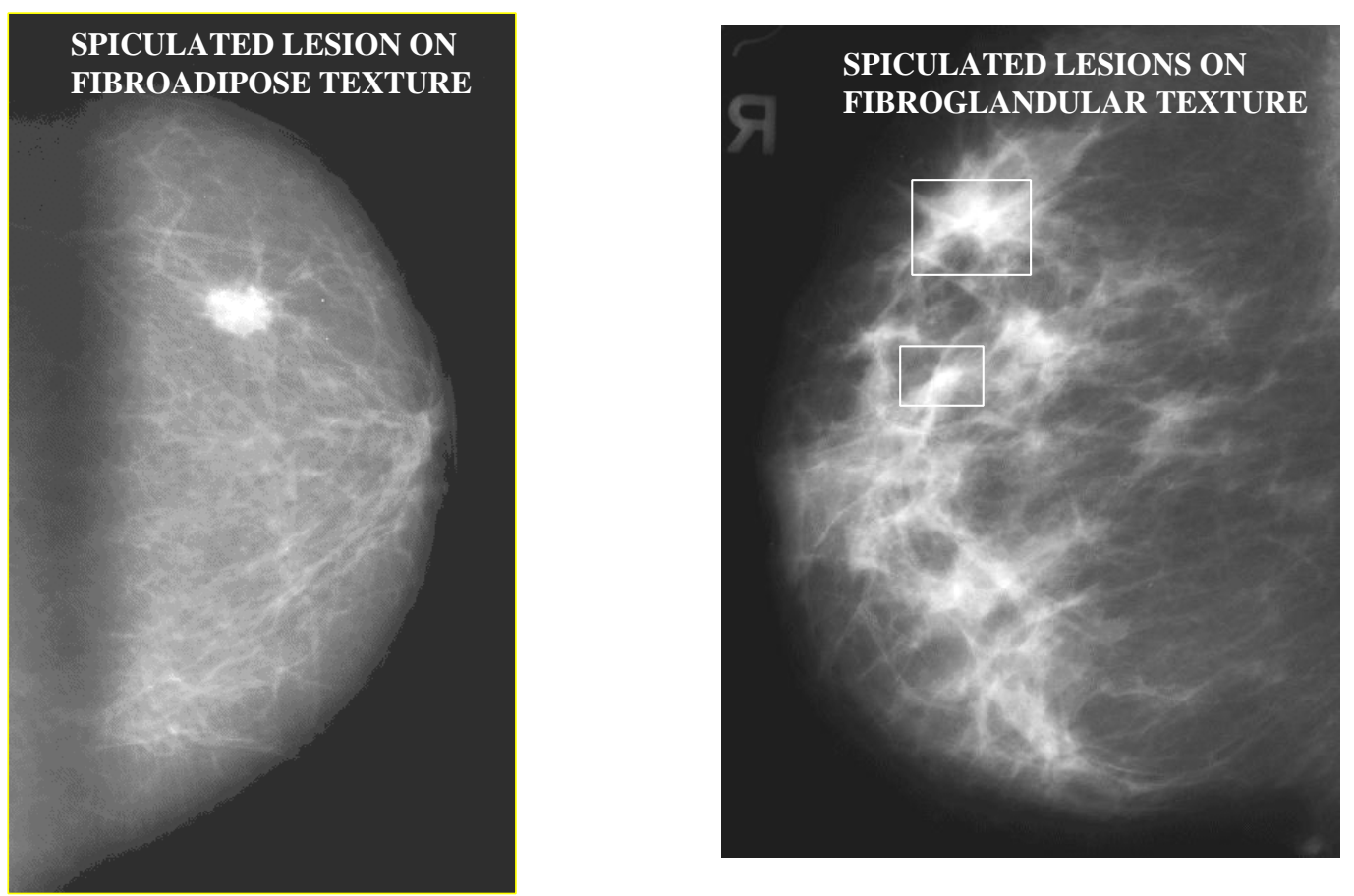

Figure 2 - The same type of lesion on different background: Adipose (left) and Glandular (right).

Figure 3 reproduces one prototype for each of the six classes found by radiological experience. Texture analysis was made using three different approaches, with non supervised neural networks or with a mixed approach (features extraction combined with FF-ANN [3]).

Using a 2-D Fourier Transform approach, an inner region to the mammogram is selected and on this it proceeds with an analysis in frequency, choosing the values that characterize the several classes.

Using another approach called statistical method, we have built a medium image for every class (for a total of six) and for every image we calculate the average and the standard deviation of the distance from every class (12 variables) to which the age of the patient is added.

Finally a Sanger Neural Network has been constructed, that it automatically extracts the more meaningful parameters for the classification, providing a Principal Component Analysis.

All the three methods 


\begin{tabular}{|l|c|c|c|}
\hline & Class. DN (\%) & Class. FA (\%) & Class. Glandular (\%) \\
\hline Fourier Transform & 100 & 67 & 93 \\
\hline Statistical approach & 98 & - & - \\
\hline Sanger Neural Network & - & 80 & 88 \\
\hline
\end{tabular}

Table 1 - Results of the texture analysis, using mixed approach and non-supervised neural networks, performed on three classes: dense, adipose and glandular textures.
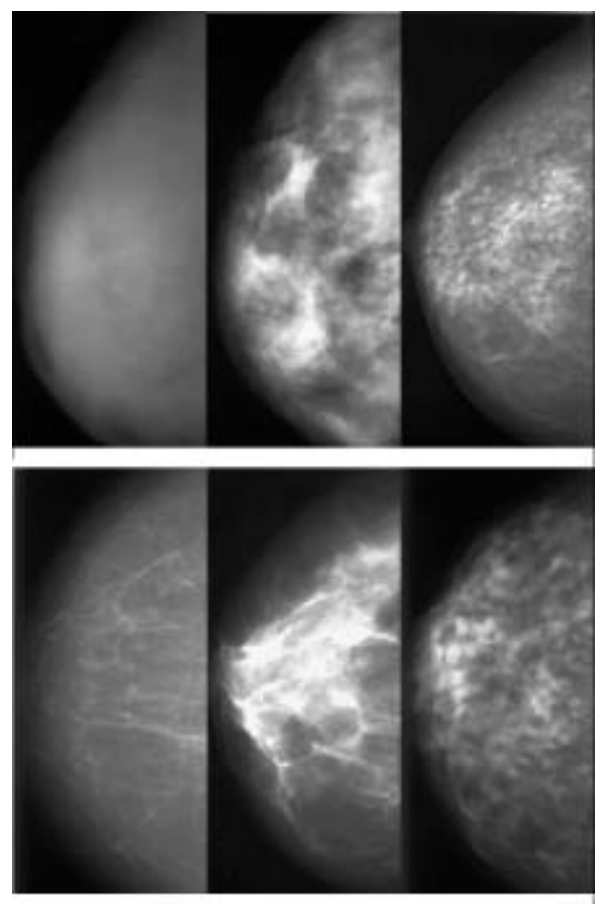

Figure 3 - From left to right and from top to bottom: dense (DN), irregularly nodular (IN), micronodular (MN), fibroadipose (FA), fibroglandular (FG), parvinodular (PN). The large class Glandular is defined as IN or MN or FG or PN

show results not definitive, but promising. In table 1 the results, discriminating between dense, adipose and glandular textures, are reported.

\section{Spiculated lesions analysis}


Massive lesions are rather large objects to be detected, but they show up with a faint contrast

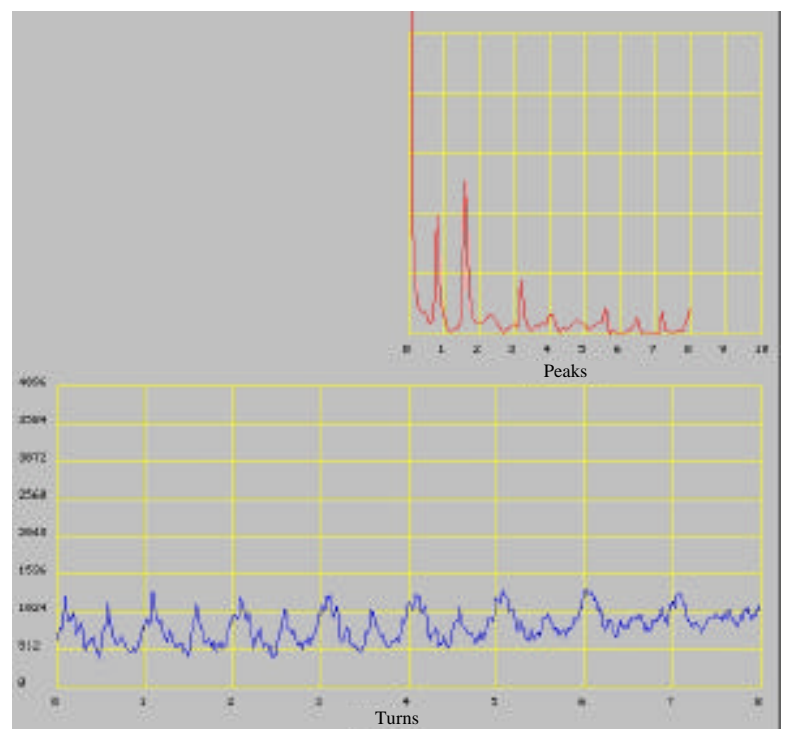

Figure 4 - Examples of graphical output from SPIRAL algorithm: bottom counts vs turns, top right corresponding Fourier Transform vs number of peaks.

slowly increasing with time. The need for tools able to recognize such a lesion at an early stage is therefore apparent. We show here our work to select the spiculated lesions, i.e. a particular kind of massive lesions looks like a star. We have written an algorithm, called SPIRAL, able to select, inside the mammogram, the Regions Of Interest (ROI) which can include a spiculated lesion. Every 10 pixels of the image a spiral, with 30 pixels inner radius and 100 pixels external radius, is calculated. For every turn 64 points, uniform angular distributed, are selected to built a vector of 512 (8 turns). A Fourier Transform $F(k)$ calculation is made. In figure 4 the graphical output from the SPIRAL algorithm is shown on a region containing a spiculated lesion. We calculate the integral of the $\mathrm{F}(\mathrm{k})$ from $\mathrm{k}_{1}$ to $\mathrm{k}_{2}$ peaks and built a Fourier Map, whose values are proportional to the probability to be close to a spiculated lesion. The ROI are composed from the points of the image whose values, in the Fourier Map, are over a fixed Fourier Map Threshold (FMT). Figure 5 shows the results obtained integrating $\mathrm{F}(\mathrm{k})$ from 1 to 3 peaks in terms of the sensibility for patient and average areas vs FMT. 


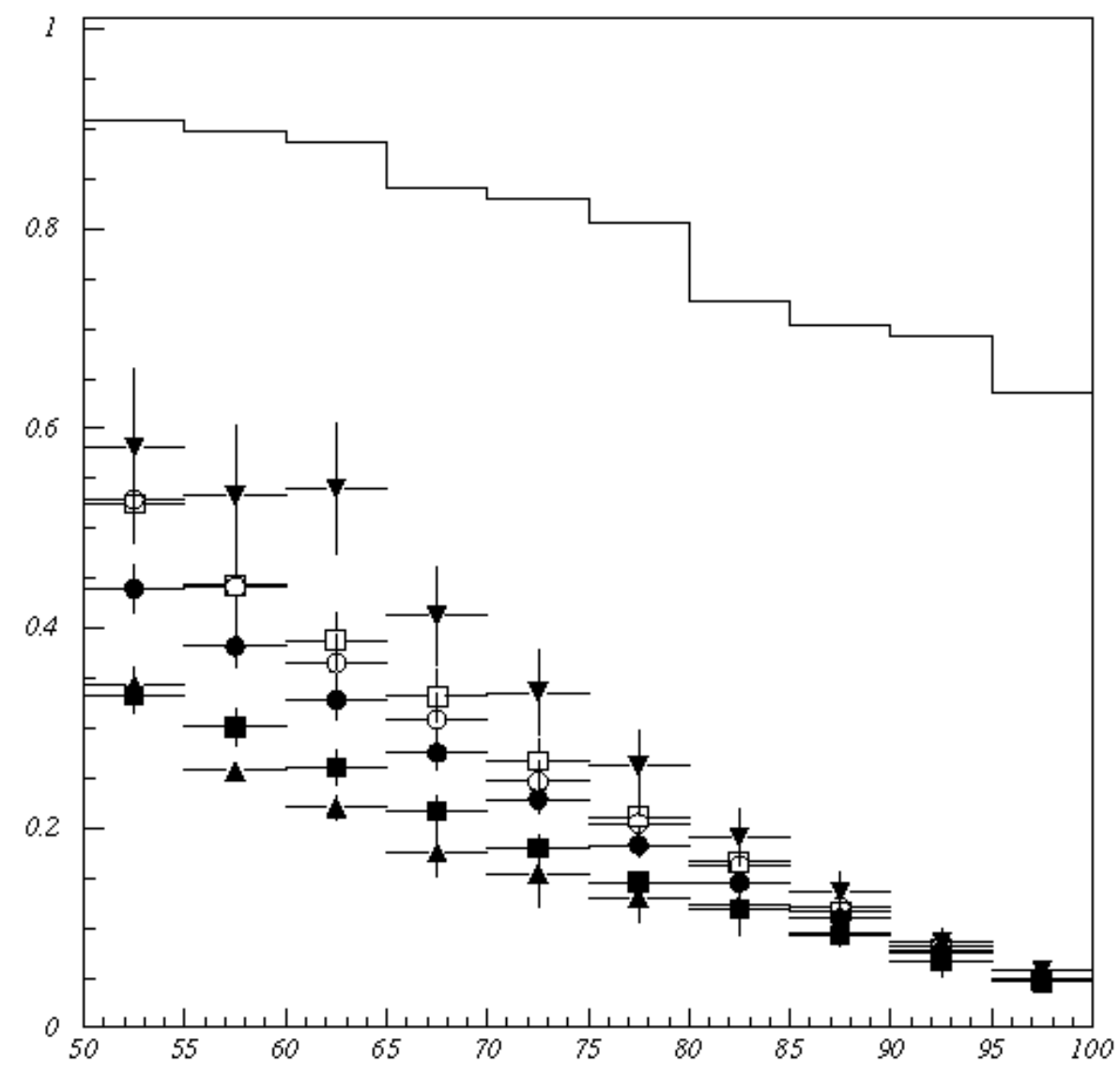

Figure 5 - Global results of SPIRAL algorithm. The curve is the sensitivity for patients versus FMT. The points are average areas of ROIs normalized to $100 \mathrm{~cm}^{2}$ for the six classes of textures: up side black triangles are DN, empty squares are PN, empty circles are IN, black circles are FG, black squares are FA and down side black triangles are MN.

Fixing FMT at $75 \%$, the number of false positives is of the order of 1.4 per image keeping an $85 \%$ sensitivity on our sample.

\section{Microcalcification clusters analysis}

A microcalcication is a rather small $(0.1$ to $1.0 \mathrm{~mm}$ in diameter) but very brilliant object. Some of them, either grouped in cluster or isolated may indicate the presence of a tumor. In figure 6 some examples of microcalcifications clusters are shown. Microcalcification cluster analysis was made using two different approaches:

features extraction combined with Feed-Forward Neural Network (FFNN);

statistical study of the higher order moments of the histograms of extracted windows from the image. 

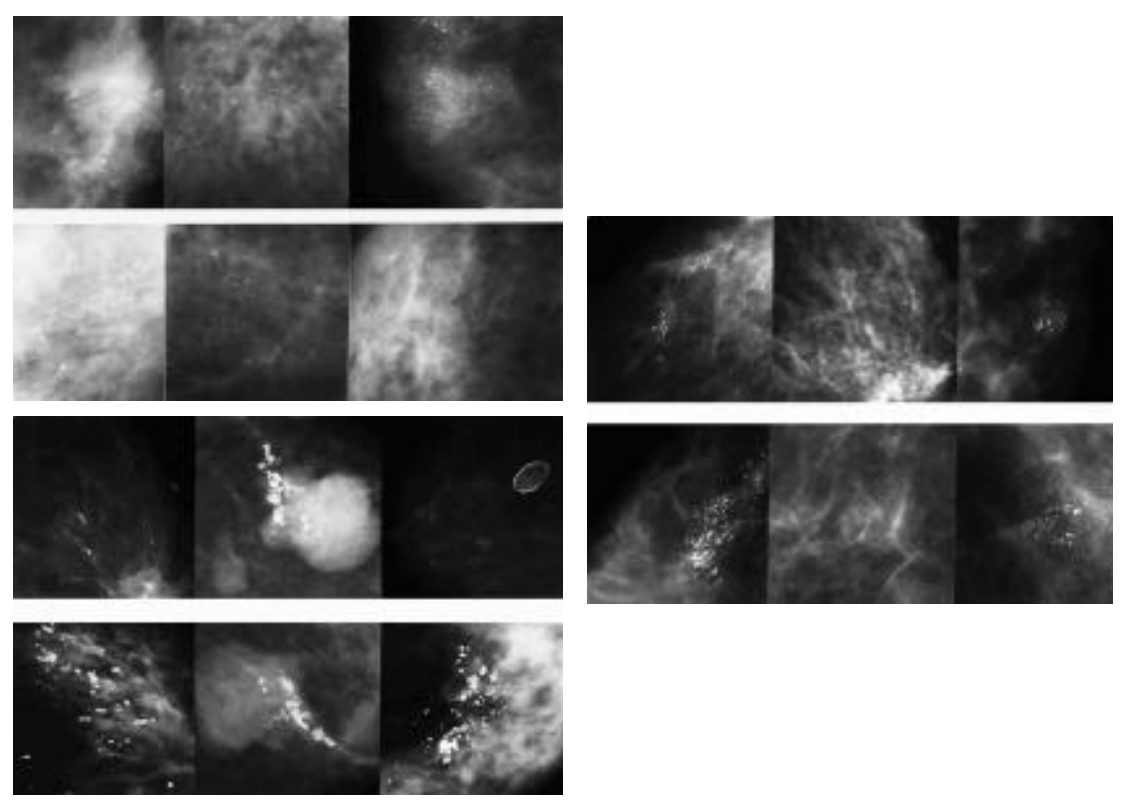

Figure 6 - various examples of microcalcification clusters

In the first approach masking is used, to eliminate low spatial frequency fluctuations and enhance spikes, without introducing artifacts. High pass linear filters with different kernel dimension and convolution masks are used. From both original and processed window features are extracted and used as inputs for a FFNN. These input variables are absolute maximum intensity, average, variance, number of local maxima, minimum and maximum of the distances between maxima, average distance between maxima, average surface of spikes and average intensity of local maxima. The method optimization finds the better choice for filtering, for neural network (18 input neurons, 2 hidden neurons and 1 output neuron) and learning algorithm (Back Propagation with Momentum). The final results show a sensitivity of $86 \%$ and specificity $90 \%$. In the second approach on the processed window the Skewness (S) and Kurtosis (K) are calculated. The presence of the microcalcification clusters produces asymmetries into the histogram of the filtered window. The classification rule shows microcalcification clusters occur if $S>1$ and $K>2$. The final results show a sensitivity of $93 \%$ and a specificity $79 \%$.

\section{Conclusions}

The CALMA collaboration has acquired, currently, the European largest database of mammographic images. Using this database was been possible to verify the validity of the ROI hunter procedures for the spiculated lesions and the microcalcification clusters. The achieved results are close to the human performance and it is possible, starting from these results, to develop CAD tools to be used as a second radiologist in the classification of breast diseases. 


\section{References}

[1] A.G.Haus and M.J.Yaffe, Editors, A categorical course in physics. Technical aspects of breast imaging. Radiological Society of North America, 1993, presented at the 79 th scientific assembly and annual meeting of RSNA.

[2] R.A.Smith, "Epidemiology of breast cancer", in "A categorical course in physics. Imaging considerations and medical physics responsibilities", Madison, Wisconsin,1991. Medical Physics Publishing.

[3] A.Zell et al., "SNNS - Stuttgart Neural Network Simulator - V4.1", University of Stuttgart Report No.6/95 (1995) 